\title{
Cancrum Oris-associated with Acute Myeloid Leukemia: A Forgotten Disease
}

\author{
${ }^{1}$ Arvind Krishnamurthy, ${ }^{2}$ Anita Vaidhyanathan, ${ }^{3}$ Satish Srinivas \\ ${ }^{4}$ Shirley Sundersingh, ${ }^{5}$ Krishnarathinam \\ ${ }^{1}$ Associate Professor, Department of Surgical Oncology, Division of Head and Neck Oncology, Cancer Institute (WIA), Adyar \\ Chennai, Tamil Nadu, India \\ ${ }^{2}$ Observer, Department of Surgical Oncology, Division of Head and Neck Oncology, Cancer Institute (WIA), Adyar, Chennai \\ Tamil Nadu, India \\ ${ }^{3}$ Assistant Professor, Department of Radiotherapy, Division of Head and Neck Oncology, Cancer Institute (WIA), Adyar, Chennai \\ Tamil Nadu, India \\ ${ }^{4}$ Associate Professor, Department of Pathology, Division of Head and Neck Oncology, Cancer Institute (WIA), Adyar, Chennai \\ Tamil Nadu, India \\ ${ }^{5}$ Professor, In Charge of Hematology, Division of Head and Neck Oncology, Cancer Institute (WIA), Adyar, Chennai \\ Tamil Nadu, India
}

Correspondence: Arvind Krishnamurthy, Associate Professor, Department of Surgical Oncology, Division of Head and Neck Oncology, Cancer Institute (WIA), 36, Sardar Patel Road, Adyar, Chennai-20, Tamil Nadu, India, Phone: 9840448174 e-mail: drarvindkrishnamurthy@yahoo.co.in

\begin{abstract}
Cancrum oris is an orofacial gangrene, which during its fulminating course causes, progressive and mutilating destruction of the infected tissues with a consortium of microorganisms. This condition is considered to represent the "face of poverty" because factors connected with poverty, such as chronic malnutrition, poor oral hygiene and sanitation, faecal contamination, and exposure to viral and bacterial infections in an immunosupressed host contribute to disease progression. This condition is seen almost exclusively among the young children and carries a high mortality rate.

We present a case of cancrum oris in a 45 years old lady being treated for acute myeloid leukemia with chemotherapy, who in addition to a polymicrobial bacterial infection had superinfection with Mucormycosis.
\end{abstract}

Keywords: Cancrum oris, noma, leukemia, mucormycosis.

\section{INTRODUCTION}

Cancrum oris (noma from the Greek nomein “to devor") is a rapidly progressive, polymicrobial, opportunistic infection, the end result of which is spontaneous necrosis of the mucus membrane of the oral cavity. Common predisposing diseases in the past as well as present include typhoid, measles, diphtheria, leukemia, bronchopneumonia, leukemia, AIDS, tuberculosis, and whooping cough. ${ }^{1}$

Clinically, a characteristic foul odor often constitutes the initial evidence of the disease. Oral examination may show a painful red or purplish red spot or an indurated papule on the oral mucous membrane. This rapidly breaks down to form an ulcer. Thus, the oral mucosa may be affected by necrosis and ulceration, forming characteristic "punchedout" areas. The ulcerated area rapidly diffuses to adjacent and contiguous tissues, with little or no evidence of body mechanisims. Adjacent tissues first become indurated with a wax like shine. Subsequent signs of diffuse edema and erythema, a sore mouth, painful lip or cheek, bluish tissue discoloration, profuse salivation, and purulent discharges from the mouth or nostril may ensue. As the disease progresses, exfoliation of the teeth often occurs as a result of destruction of the supporting alveolar bone. Sequestration of this exposed bone is common. Cervical lymphadenopathy in most cases is quite pronounced, but it varies with the severity of the condition. Other clinical manifestations include fluctuating fever, general malaise, increased cardiac and respiratory rates, and moderate to severe localized pain. ${ }^{2}$

We report a case and discuss the surgical management of cancrum oris complicating an adult lady who was undergoing chemotherapy for acute myeloid leukemia.

\section{CASE REPORT}

A 45 years old lady with fever and cough for a month's duration was evaluated at a local hospital for lower respiratory tract infection. The presence of atypical cells in the differential leukocyte count prompted a suspicion of acute leukemia and she was referred to our center for further 
management. There was no history of any abnormal bleeding episodes. On further evaluation, a bone marrow aspiration and biopsy revealed 70 to $80 \%$ blast cells, flow cytometry showed acute myeloid leukemia with an immunophenotype M1. Cytogenetic study revealed a normal karyotype and her serum did not harbor FLT3 mutation. A chest skiagram showed a resolving right pneumonic patch. Her electrolytes, uric acid, renal, hepatic and coagulation parameters were within normal limits. She was diagnosed to have acute myeloid leukemia M1 intermediate risk and she was initially started on induction Mitoxantrone and Cytarabine.

She was planned for a second course of induction after the bone marrow on day 22 of the first induction was suggestive of residual disease.

From day 7 of the second induction, she had persistent fever with weakness and malaise which persisted despite broad spectrum antibiotics. On day 16, she developed a punched out ulcer in the left buccal mucosa. The foul smelling pus discharge from the ulcer culture had grown moderate growth of Pseudomonas aeruginosa, Staphylococcus aureus and Klebsiella. The infection flared up within a couple of days and quickly progressed to full thickness gangrene of the entire left cheek, sparing the mandible and maxilla (Fig. 1). She was taken up for wound debridement on day 19 of the second induction under the cover of broad spectrum antibiotics and antifungals (Fig. 2). The leukocyte and platelet counts had normalized and the bone marrow aspiration was reactive by them. The histopathology of the debrided tissue was suggestive of chronic necrotising fasciitis; in addition mucormycosis was

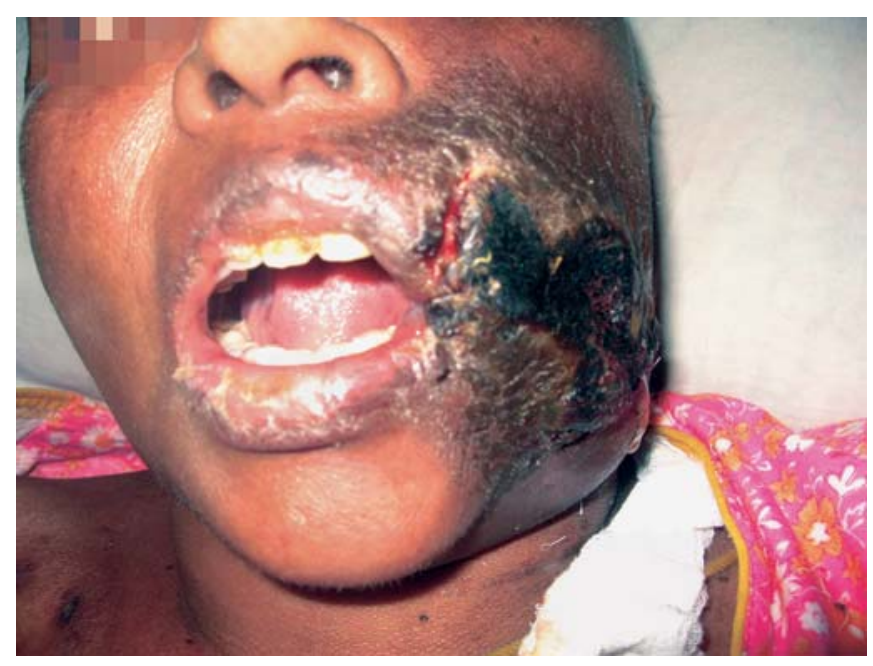

Fig. 1: Full blown cancrum oris

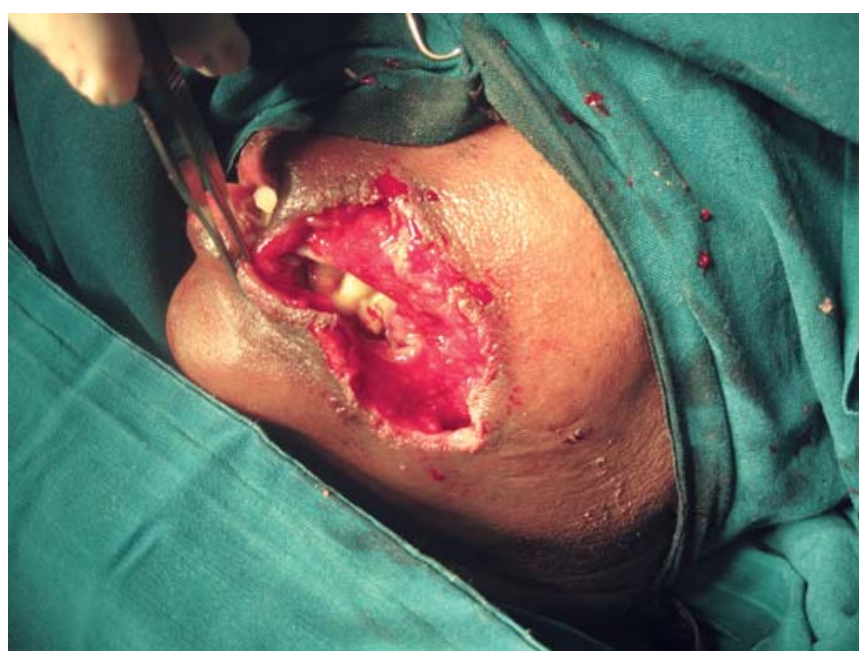

Fig. 2: After aggressive debridement

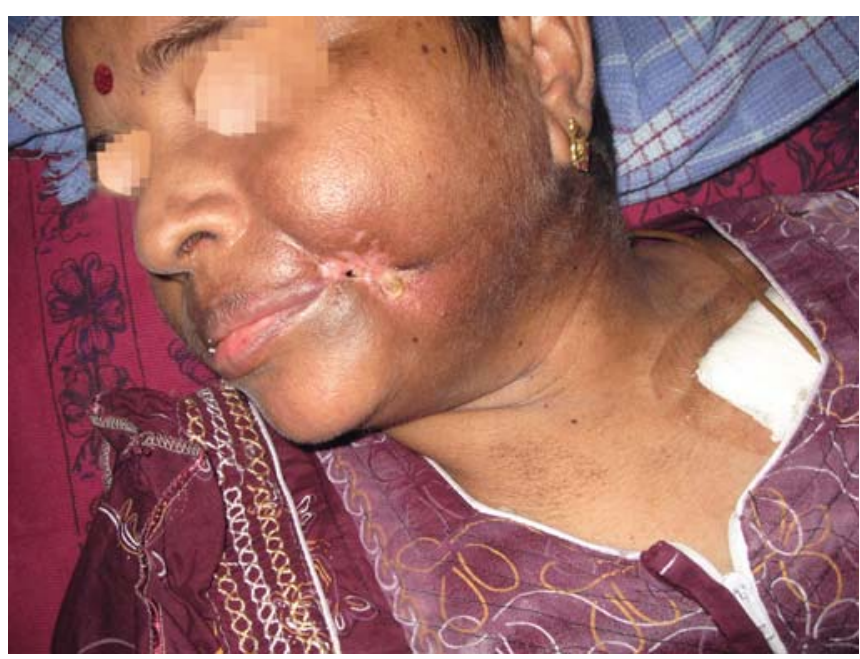

Fig. 3: Wound healing after local advancement

also demonstrated with classical vascular invasion of the fungal hyphae.

The resultant soft tissue defect was managed by daily dressings, appropriate broad spectrum antibiotics, Amphotericin B and local advancement of the tissues was done after a month to aid in complete healing (Fig. 3). The patient was taken up for consolidation chemotherapy and is now in remission.

\section{DISCUSSION}

Cancrum oris, also known by names, such as Noma and Gangenous Stomatitis was first described by Tourdes in 1848. It has also been described in the Nazi concentration camps during World War II. ${ }^{3}$ The epidemiology of noma 
has not changed much over the years, except that there has been a reduction in the mortality rate from $90 \%$ to about 8 to $10 \%$, mainly because of modern antibiotics. ${ }^{4,5}$

It is difficult to pinpoint the specific trigger agent in the complex microbiota of a noma lesion. It has been speculated that Borrelia vincentii and Fusobacterium are prominent bacteria in such lesions. ${ }^{6,7}$ Also, symbiotic relationships between fusiform bacilli and nonhemolytic Streptococci and Staphylococci have been considered significant factors in the development of noma. ${ }^{2}$ Previous studies were hampered by a lack of specimens from early stages of the lesion, confusing microbial taxonomy, lack of animal models for studies and difficulty in culturing microorganisms. ${ }^{6}$ Some reports have suggested that these microorganisms are resistant to penicillin, ${ }^{7}$ which emphasizes the need for culture and sensitivity tests before administration of antibiotics. Also, besides contemporary literature, the disease seems to possess a multifactorial etiology, so additional studies are warranted to elucidate its exact microbiology. The complex microbiota in our case was represented by the following bacteria, i.e. Pseudomonas aeruginosa, Staphylococcus aureus and Klebsiella, no fungus was seen on cultures. However, the necrotic tissue sent for histopathology demonstrated mucormycosis with vascular invasion.

The management of cancrum oris is extremely challenging and preferably done by a multidisciplinary team. Early aggressive surgical debridement is the key to a successful outcome, as was done in our case along with other supportive measures like correction of dehydration, nutrition by Ryles tube feedings, maintenance of oral hygiene and antibiotics as deemed appropriate along with Amphotericin B.
It is recommended that consideration needs to be given for correction of the resultant facial deformities only after the acute event has subsided. Reconstruction is also challenging at times requiring myocutaneous or osteomyocutaneous flaps. The wound healing in our case was aided by daily dressing and delayed local advancement of adjacent tissues.

\section{CONCLUSION}

Cancrum oris (noma) is practically a forgotten disease. It is of interest as a head and neck surgeon is rarely called upon to treat noma, a disease for which early diagnosis and prompt intervention greatly influences the final outcome.

\section{REFERENCES}

1. Weinstein RA, Choukas NC, Wood WS, MaywoodI I. Cancrum oris-like lesion associated with acute myelogenous leukemia. Oral Surg Oral Med Oral Pathol Jul 1974;38(1):10-14.

2. Berthold P. Noma: A forgotten disease. Dent Clin North Am 2003;47(3):559-74.

3. Adolph HP, Yugueros P, Woods JE. Noma: A review. Ann Plast Surg 1996;37:657-68.

4. OJI C. Cancrum oris: Its incidence and treatment in Enugu, Nigeria. Br J Oral Maxillofac Surg 2002;40(5):406-09.

5. Marck KW. A history of noma, the "Face of Poverty." Plast Reconstr Surg 2003;111(5):1702-07.

6. Falker WA, Enwonwu CO, Idigbe EO. Isolation of Fusobacterium necrophorum from cancrum oris (noma). Am J Trop Med Hyg 1999;60(1):150-56.

7. Falker WA Jr. Enwonwu CO, Idigbe EO. Microbial understanding and mysteries of noma (cancrum oris). Oral Dis 1999;5(2):150-55. 